\title{
Wisdom of Crowds for Reliable Discussion and Need Finding: A Case Study of Information Sharing Regarding Radiation after the Fukushima Nuclear Disaster
}

\author{
Yang Ishigaki \\ The University of Electro- \\ Communications \\ pokega@tanaka.is.uec.ac.jp
}

\author{
Yutaka Matsuno \\ Nihon University \\ matsuno.yutaka@nihon-u.ac.jp
}

Kenji Tanaka

The University of Electro-

Communications

tanaka@is.uec.ac.jp

\author{
Koichi Bando \\ The University of Electro- \\ Communications \\ bando@tanaka.is.uec.ac.jp
}

\begin{abstract}
This action research adopts a case study approach of reliability assurance and need finding for radiation monitoring on social media communication through the development and use of Pocket Geiger (POKEGA), a smartphone-connected radiation detector developed after the Fukushima Nuclear Disaster in Japan. We also facilitated the use of an inclusive Facebook community for radiation monitoring established by volunteer experts and normal users. Interaction through this social media led to credible discussions and enabled dose verification among users. It is important to grasp, at least roughly, the potential needs of and/or information required by users, especially those living in and around affected areas, from among the numerous topics posted by regular citizens and radiation experts on social media. Therefore, we developed a Web service called Crowd Talks to summarize and visualize discussions within the POKEGA community. This tool provides quick analysis and visualization of discussion topics from POKEGA Facebook posts based on natural language processing algorithms such as MeCab, latent Dirichlet allocation, and principal component analysis. Our results suggested that Crowd Talks was able to identify major topic clusters from the Facebook community.
\end{abstract}

\section{Introduction}

In crisis communication, social media is a useful tool for grasping the situation of and understanding the needs in affected areas; however, it remains problematic in terms of reliability assurance and the ability to summarize posts. Reliability assurance is vital for avoiding misinformation or rumors, which tend to spread rapidly on social media. Since the information shared on social media is substantial, official bodies or disaster volunteers need a data digest in order to acquire appropriate information from affected areas, such as the post-disaster situation and necessary supplies, in a timely fashion.

In our previous action research, we developed a series of inexpensive yet accurate mobile radiation detectors, which we called Pocket Geiger (POKEGA), in order to address the urgent desire of ordinary people to measure and share radiation levels in their milieus and discuss the current situation and outcomes of the 2011 Fukushima Nuclear Disaster in Japan in a Facebook community [1]. In Section 2 of this paper, we summarize the involvement of POKEGA in the social community based on our previous study, and in subsection 2.4 we discuss the reliability of crowd communication from the aspect of inter-domain discussions between ordinary people, radiation experts, and the public at large.

Although thousands of posts comprising fruitful discussions between users and experts were made in the POKEGA community, we were unable to track all of the discussions and grasp the primary needs of users in terms of nuclear disaster communication. Therefore, in 2016, in order to summarize and visualize these discussions, we developed a Web service called Crowd Talks [2]. This tool enables Facebook posts to be captured, analyzed using natural language processing algorithms such as MeCab and latent Dirichlet allocation (LDA), and then visualized using principal component analysis (PCA). In Section 3, we discuss the application of Crowd Talks to the POKEGA Facebook community. 


\section{Reliable crowd discussion}

POKEGA is a radiation detector that connects to $\mathrm{iOS} \circledast$ or Android® smartphones without the need for any modifications (Figure 1). It uses Global Positioning System (GPS) and networking capabilities to log and share data in Facebook group discussions. To reduce costs while maintaining accuracy and flexibility, we used a combination photodiode detector and microphone interface.

PIN photodiodes can detect nuclear radiation in the depletion layer [3]. However, to our knowledge, our project marks the first time a photodiode of this type has been combined with a general commercial smartphone in a practical manner. The detector circuit design is optimized for cost and simplicity, while the smartphone software application is tasked with handling the required complex processing [4].

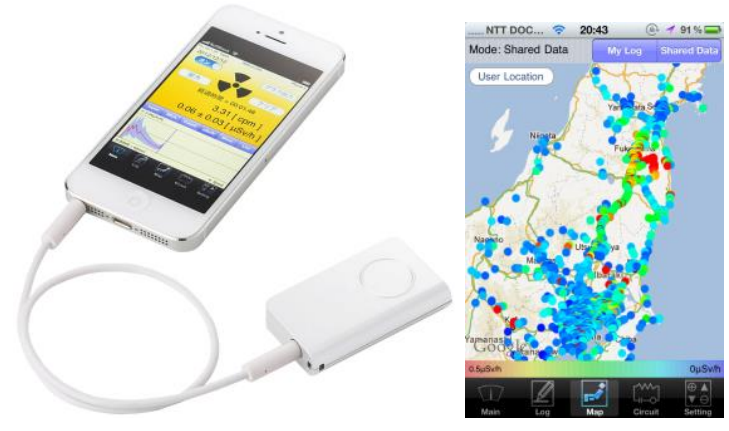

Figure 1 Pocket Geiger device and software

\subsection{Start-up phase}

We designed POKEGA based on general-purpose semiconductors under open source licensing before immediately launching the development project on a website, which we registered under the name Radiation-Watch.org, to gather volunteer collaborators from around the world. We also listed our project on the crowdfunding website Kickstarter.com to promote quicker and more cooperative development. As a result, the project received pledges from 167 backers in 23 countries. We also received unexpected offers of assistance from hackers and professional researchers in the form of technical advice, calibration testing, and field experiments. These achievements demonstrate the effectiveness of crowdfunding not only for securing financing, but also for attracting publicity and experts sympathetic to the goals of the project.

Two Dutch specialists in the Netherlands separately and voluntarily offered to calibrate the POKEGA device in their specialized facilities. Their examination was extremely useful because it revealed that the measurement range for Cesium-137 $\left({ }^{137} \mathrm{Cs}\right)$ made possible by a POKEGA-equipped smartphone was approximately 0.05 to $10 \mathrm{mSv} / \mathrm{h}$ [5], which covers most radiation levels measured in Japan. In an interview in a Dutch newspaper [6], the specialists stated that they had originally discovered the project on Kickstarter and decided to support it since they deemed it a worthwhile endeavor.

A Japanese researcher in the field of soil physics also offered to examine the performance of the device in an outdoor environment in an evacuation area in Fukushima Prefecture. Along with colleagues, he constructed field monitoring systems that included POKEGA in three different vegetation areas: a garden, a forest, and a deforested area [7].

Based on the results of these experiments, realistic problems involving false detection were apparent in two error modes. One error mode occurred on days with abundant sunlight because the near-infrared light sometimes penetrated the shade tape inside the POKEGA case. The other error mode occurred on warm days because at a temperature of approximately $40^{\circ} \mathrm{C}$ or higher, thermal noise generated a false signal in the photodiode sensor. As countermeasures for these problems, we improved the performance of the shade tape and reconfigured the parameters of the circuit to set higher thresholds for the radiation signal and prevent false detection.

Other experts or hackers also actively contributed to POKEGA development by providing rich technical advice from laboratory experiments or technological suggestions regarding circuit design and smartphone software. As a result of such collaborative research, POKEGA has evolved through six different designs. For example, the latest design is 10 times more efficient than the original version in detecting gamma radiation.

\subsection{Collaboration phase}

As of this writing, approximately 50,000 POKEGA units had been distributed and more than 2,500 subscribers had joined the Facebook community, where they could report measurement results and discuss radiation protection or technical improvements. For example, as shown in Figure 2A, within the community, children were advised to avoid a children's park through the use of a poster and a bluecolored sheet that covered contaminated soil because the background radiation level in the area was 0.49 $\mu S v / h$, compared with the normal level in Tokyo of around $0.05 \mu S v / h$. Figure $2 \mathrm{~B}$ shows a photo taken in a prohibited area around a frontline base called J-Village, where radiation levels ranged from $0.45-1.90 \mu \mathrm{Sv} / \mathrm{h}$. 
The poster advised users to return home until administrative approval to be in the area was granted. Through data sharing, people learned about radiation levels in various locations, including places they did not typically go.

We released six versions of POKEGA as we improved various technical specifications of the device, including sensor efficiency, internal power generation, noise reduction, visualization features, and support for various mobile devices. Most of these improvements were inspired by technical proposals on the POKEGA Facebook page from volunteer engineers and radiation professionals working with our open source licensing.

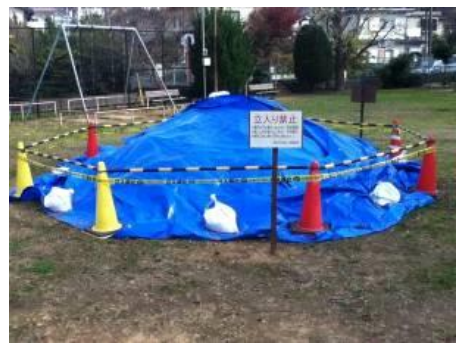

A. Children's park

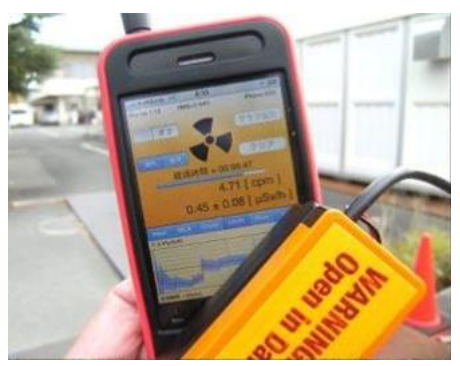

B. The J-Village in Fukushima

Figure 2 Photos taken and posted by users

\subsection{Mutual verification phase}

Numerous users have compared readings from POKEGA with those from other calibrated dosimeters, including monitoring stations constructed by the Japanese Government. For example, in Figure 3A, the results of comparative tests with an official monitoring station in the Fukushima area a few months after the nuclear accident are shown. Since POKEGA produced nearly the same readings as other detectors in most cases, it gained the trust of users. In contrast, some people commented that they had changed their mind about the government-issued instruments due to a lack of trust.

In Figure 3B, interaction is shown between users and radiation professionals that originated from a question posed by a business traveler. The business traveler found that the level of natural background radiation in Okayama Prefecture was slightly high $(0.13 \mu \mathrm{Sv} / \mathrm{h})$, even though it is more than $600 \mathrm{~km}$ away from Fukushima. Subsequently, another user reported that they had obtained a nearly identical reading in Fukushima. Following this exchange, a radiation specialist noted that, based on official local government data, the average natural background radiation level in Okayama before the nuclear disaster was around $0.126 \mu S v / h$ because of the geological properties of the area. In this case, three POKEGA users, including a radiation expert, quickly and adequately answered the question within 4 days of the first post.
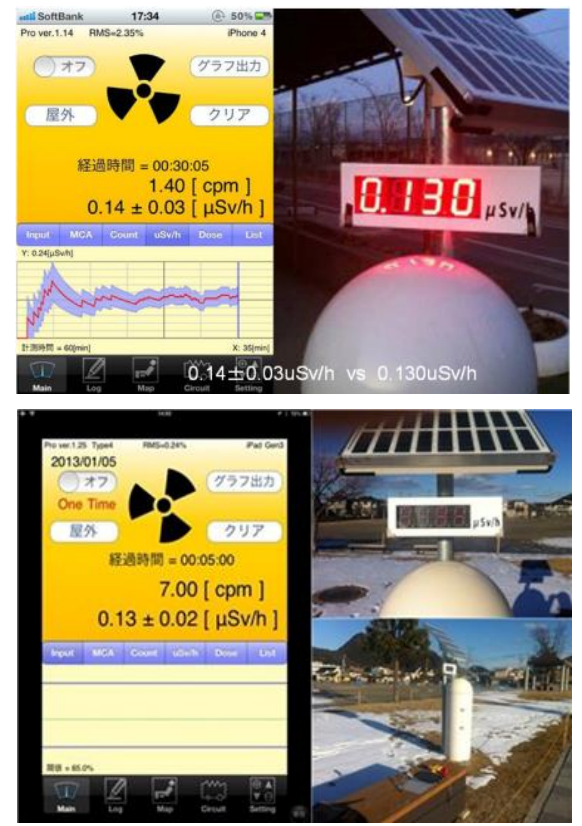

A. Comparative tests in Fukushima

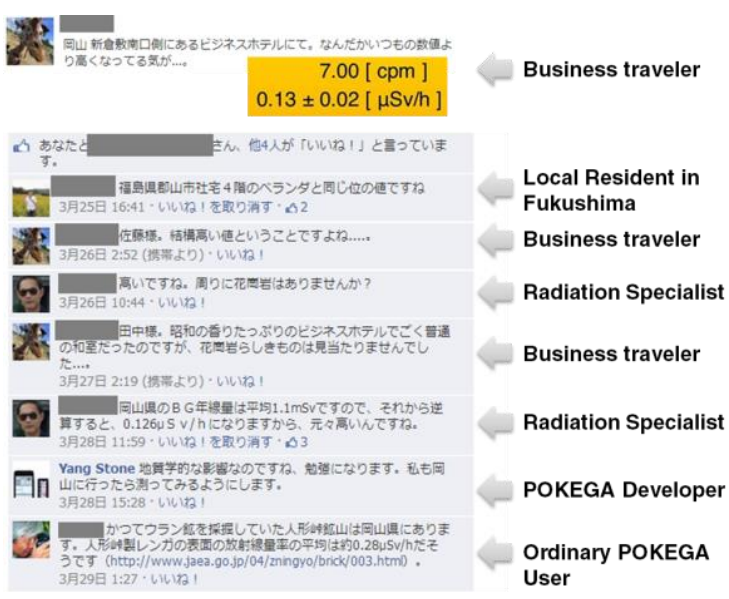

B. Problem solving regarding radiation level

Figure 3 Example of social interaction 


\subsection{Dynamics of credible discussion}

Turoff et al. reported the effectiveness of unofficial, user-generated information, e.g., "back-channel information" or the "wisdom of crowds", generated by ordinary people through social media in crisis communication [8]. However, they also indicated the risk of misinformation or overconfidence (or overdiffidence) and the difficulties involved in information sharing with official bodies. In the following section, we model the dynamics of credible discussion by social inclusion on POKEGA's community in order to discuss solutions for these problems.

In Figure 4, the dynamics of POKEGA development are proposed between two major stakeholders: ordinary users and radiation experts. In the start-up phase (Figure 4A), users and experts separately joined and contributed to project development as they individually backed and publicized the Kickstarter campaign by re-tweeting or sharing information about the site on the POKEGA Facebook page; some experts even offered to provide voluntary examinations.

In the collaboration phase (Figure 4B), the stakeholders began bottom-up collaborations in which users started sharing radiation levels in their milieus and experts started reporting test results and technical information based on the device's open source licensing. Experts provided fruitful information for ordinary users through discussions in the Facebook group, for which many users expressed their appreciation. Such direct radiation risk communication was not only helpful for the users, but also effective for fostering trust among all stakeholders.

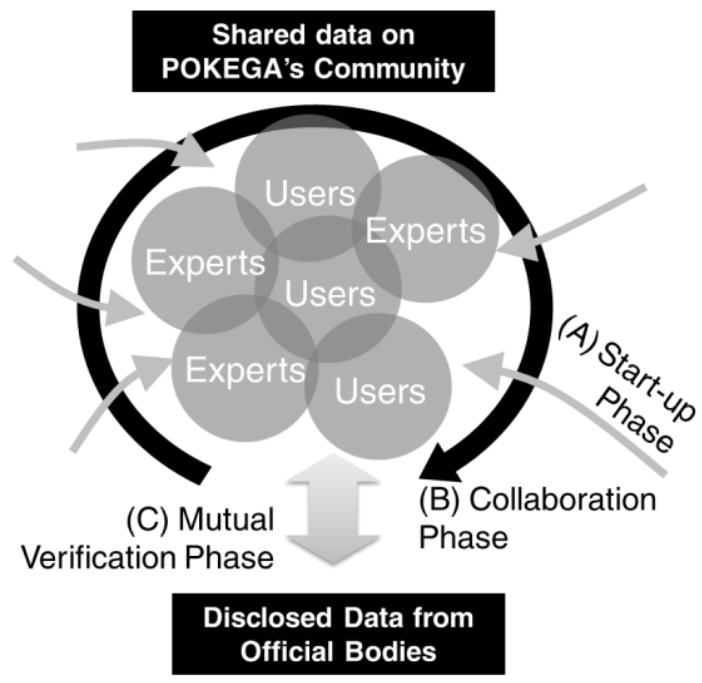

Figure 4 Interaction model of the various development phases
POKEGA also allows a comparison between its radiation measurements with official data from monitoring stations constructed by the Japanese Government, which we refer to as "mutual verification" (Figure 4C). Users can actively report comparative testing results, which can then be followed-up by experts. This style of citizen-centered development also helps foster trust among users. The mutual verification process widely ensures POKEGA's performance, reliability, and trust among users. On the other hand, after mutual verification, some users find that the data disclosed by the Japanese Government were true.

In contrast, in the "official bodies" layer, a decision-making process is needed for disclosing data, which generally takes a long time. In fact, soon after the Fukushima accident, the Japanese government knew the results of detailed simulation results from a computer-based decision support system for nuclear plant accidents, named SPEEDI (System for Prediction of Environmental Emergency Dose Information), which was developed for nuclear disaster management [9]. However, although they shared a few documents with the public two weeks after the accident because they received severe criticism, they chose not to disclose radiation dispersal data with researchers or the Japanese public for over two months [10]. Ono indicated that the risk assessors' hesitation might have been due to a lack of skill regarding when to use/disclose simulation results or how to explain errors or uncertainties [11].

To realize the "wisdom of crowds" in crisis communication situations, it is important during the collaboration phase to avoid misinformation or overconfidence in terms of the rich discussions autonomously provided by users and experts to help each other based on data, knowledge, and expertise. Furthermore, the mutual verification process shows great potential in ensuring the reliability of the device and fostering the trust of users.

\section{Analysis of social media topics}

As described in Section 2, various users, including ordinary people and radiation experts, posted thousands of topics regarding radiation protection in the POKEGA community after the Fukushima nuclear disaster. However, we had neither the time nor resources to track all of these discussions. Therefore, we needed to summarize the information contained within the POKEGA Facebook group to grasp a general idea of the potential needs of and/or information required by users, especially those living in the affected areas. 
To digest and visualize big data in social media posts, we developed a Web service called Crowd Talks that enables: 1) Facebook posts to be acquired; 2) data to be analyzed using natural language processing algorithms such as MeCab and LDA; and 3) the results to be visualized on a two-dimensional (2D) graph created using PCA mapping.

The basic implementation concept and features of the Crowd Talks tool are shown in subsection 3.1, and in subsection 3.2, we describe the use of the tool to characterize the data in the POKEGA Facebook community as a case study.

\subsection{Crowd Talks}

As shown in Figure 5, Crowd Talks consists of the following three functional blocks: cron, MongoDB, and Apache Web services (CSS, JavaScript, and PHP). In cron, a Web crawler fetches whole posts from the Facebook community specified by a user at fixed intervals. MongoDB stores the associated time stamps and HTML data, including links, photos, movies, or sensory data.
In Web services, the visualization function enables original posts in the Facebook community to be checked and a 2D graph that shows the results of topic analysis to be constructed (Figure 6). Topic analysis comprises three stages: corpus construction using $\mathrm{MeCab}, \mathrm{LDA}$, and PCA digestion. These stages are detailed as follows.

Mecab is an open source toolkit for the morphological analysis of written languages that do not have space dividers between words, such as Japanese and Korean. In Crowd Talks, MeCab extracts and counts nouns from discussions in the Facebook community to create a corpus file. We implemented two thresholds for word counting - the minimum and maximum number of occurrences - in order to ignore rare words such as proper nouns and prepositions such as "a" or "the".

LDA is a generative probabilistic model used for text modeling that is based on a three-level hierarchical Bayesian model [12]. LDA represents documents as mixtures of topics that spit out words with certain probabilities. Topic probabilities provide an explicit representation of original documents.

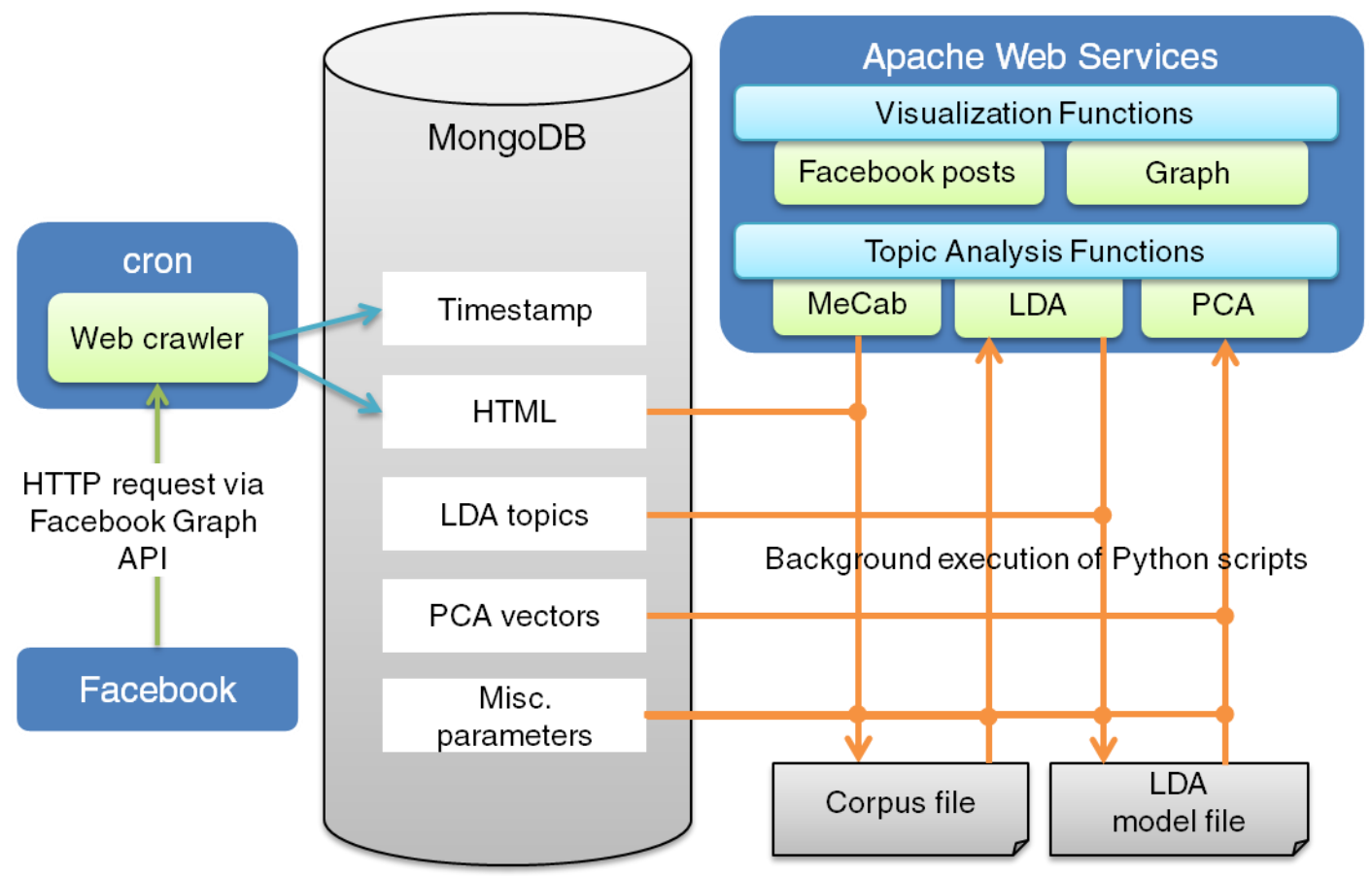

Figure 5 Functional blocks of the Crowd Talks tool 


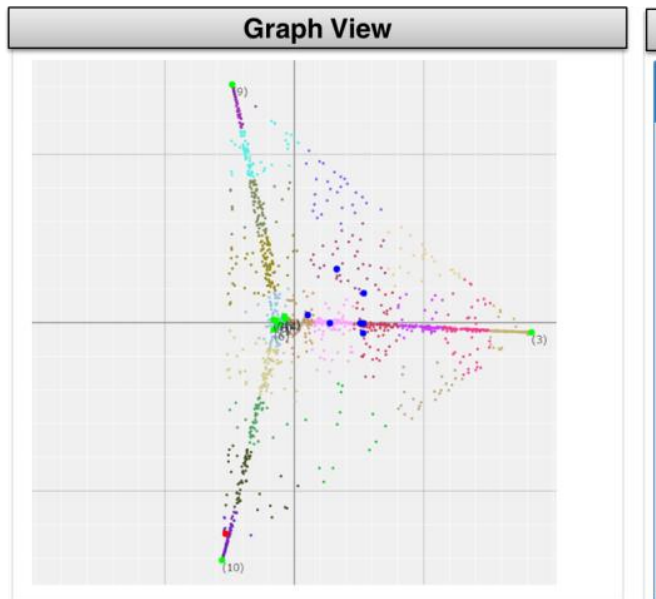

\section{LDA Topics}

(1) $=0.0208 \times[$ the $\rfloor+0.0138 \times[1]+0.0135 \times[0]+0.0132 \times[$ 湘 $]$

正 $\rfloor+0.0109 \times\lceil 0\rfloor+0.0108 \times\lceil$ and $\rfloor+0.0101 \times\lceil 4\rfloor+$

$0.0101 \times\lceil$ of $\rfloor+0.0100 \times[$ type $\rfloor+0.0086 \times$ 「よう +

(2) $=0.0197 \times[0]+0.0156 \times[の 」+0.0151 \times$ 「ボケットカイカ

-」 + 0.0114x「計測」 $+0.0085 \times$ 「1」 $+0.0083 \times$ 「さん」 +

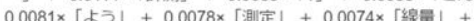

$0.0073 \times\lceil 3\rfloor+$

$(3)=0.0210 \times[0]+0.0177 \times\lceil$ 測定 $\rfloor+0.0169 \times[の\rfloor+$

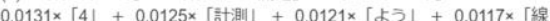

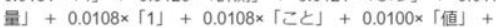

$(4)=0.0254 \times\lceil の\rfloor+0.0232 \times\lceil 0\rfloor+0.0163 \times\lceil$ 値 $\rfloor+$

$0.0154 \times$ 「版」 $+0.0153 \times$ 「測定」 $+0.0115 \times\lceil 1\rfloor+0.0113 \times\lceil 4\rfloor+$

$0.0103 \times$ 「よう」 $+0.0094 \times\lceil 5\rfloor+0.0092 \times\lceil 2\rfloor+$

$(5)=0.0290 \times[0\rfloor+0.0140 \times[の\rfloor+0.0140 \times[$ 測定 +

$0.0132 \times\lceil h\rfloor+0.0105 \times\lceil$ 線量」 $+0.0103 \times\lceil 1 」+$

$0.0098 \times[T Y P E\rfloor+0.0097 \times[\mathrm{sV}\rfloor+0.0094 \times[\mathrm{d}$.

$0.0085 \times[4]+$

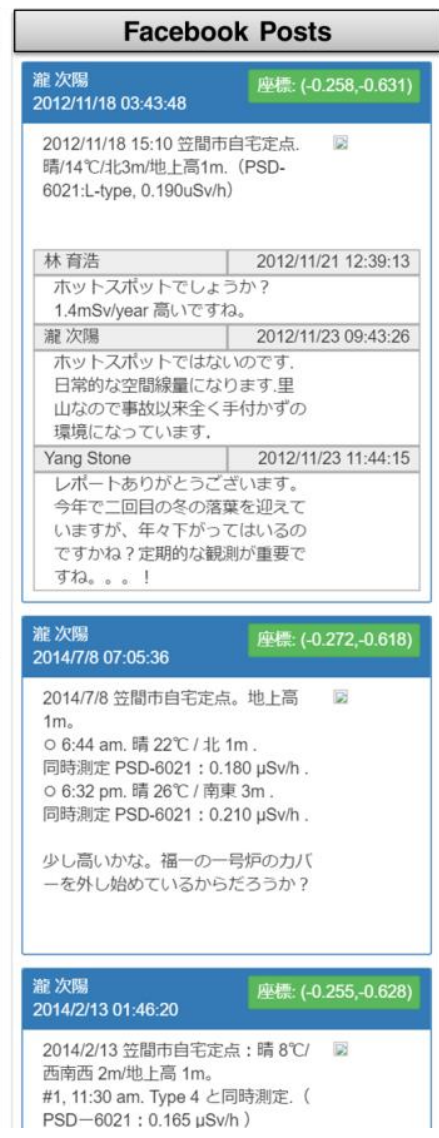

Figure 6 Dashboard layout of the Crowd Talks tool
Crowd Talks generates $n$-dimensional probability vectors for each Facebook post as follows, where $n$ is the number of LDA topics, using the Gensim library in Python.

Post $1:=\{$ Topic $1->40 \%, \ldots$, Topic $n->15 \%\}$

Post $2:=\{$ Topic $1->10 \%, \ldots$, Topic $n->45 \%\}$

PCA is then used to map probability vectors onto a readable 2D graph. Crowd Talks calculates a variancecovariance matrix and eigenvalues based on these probability vectors. Subsequently, the top two eigenvalues are chosen as the two dimensional axes for base conversion.

In a previous study, Carson et al. developed an LDA-based Web-based visualization tool called LDAvis that provides a global view of topics [13]. LDAvis allows topic-term relationships to be flexibly explored in an LDA model. However, it is unable to visualize relationships between social media posts and LDA topics.

\subsection{Need finding on POKEGA Facebook}

In this subsection, we discuss our application of Crowd Talks to the POKEGA Facebook group in order to grasp a rough idea of the potential needs of and/or information required by users, especially those living in the affected areas.

Figure 7 shows topic visualization in Crowd Talks based on 2,624 threads posted in the POKEGA Facebook group between July 2011 and July 2012. Each point represents a single post in the Facebook community. As a result of this visualization, we identified three major clusters, which are described as follows.

Cluster 1 included various discussions regarding radiation protection, such as associated health risks, hot spots, technical support for POKEGA, and natural background information, as shown in Figure 3.

Cluster 2 included the sharing of dose rates at various locations. The posts in this cluster referred to data sharing regarding radiation levels measured by mobile POKEGA users, especially around their daily 
milieus, such as children's parks, schools, farmland, or the user's own home or garden, as shown in Figure 2. Since most of the posts in this cluster included photos, we found that such rich content was important for citizens in sharing risk information.

Cluster 3 included fixed point observation reports, especially from the Fukushima area. Most of these posts did not include photos. In this cluster, users tried to observe daily trends in radiation measurements in order to check for increases or decreases.

Cluster 1 was expected because we frequently joined in Facebook discussions regarding radiation measurement or protection. Cluster 2 was also expected since we originally designed POKEGA for smartphones as a mobile radiation detector with a GPS data sharing feature. However, Cluster 3 was not expected. In Cluster 3, we found a new potential need to automatically share radiation levels at fixed locations, such as a Twitter bot for dose rates.

This new need inspired us to develop two additional items. First, we developed a new model of POKEGA referred to as Type 5 for embedded microcomputers. Type 5 devices have already been released and are currently in use at monitoring stations on DoseNet project in University of California, Berkeley [14], and also on private farms in Japan [15]. Second, we developed a data sharing protocol for radiation measurement information via Twitter for both ordinary citizens and radiation professionals. We recently started devising new guidelines regarding radiation measurement on social media [16].

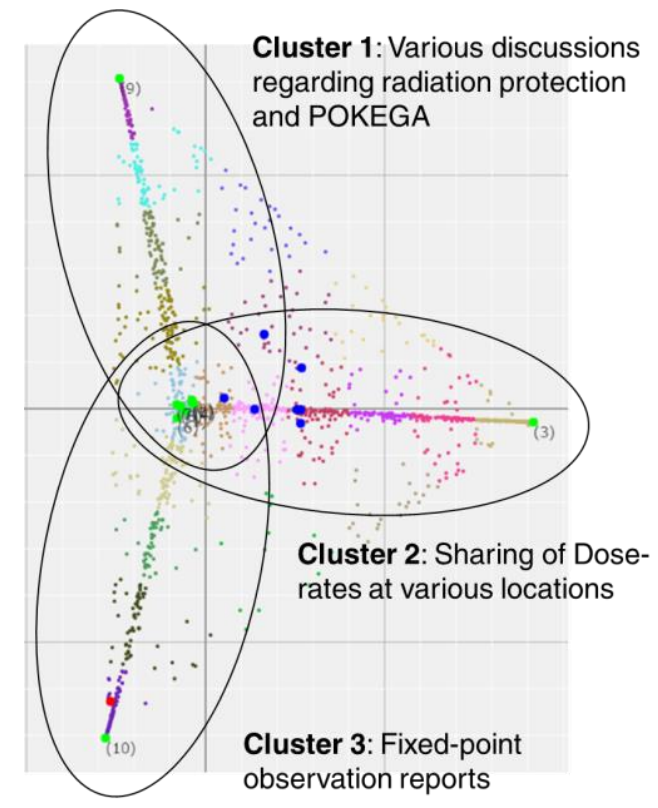

Figure 7 Visualization of topics in the POKEGA Facebook community

\section{Discussion}

Burke et al. proposed the basic concept of "participatory sensing" by smartphone devices to form interactive communities that enable public and professional users to gather, analyze, and share local knowledge for realistic social applications such as urban planning, public health, natural resource management, cultural identities, and creative expression [17]. Adopting modern communication platforms such as crowdfunding, open source development, social media, and social inclusion, this action research reported on a new pragmatic tool for radiation monitoring in situations involving a nuclear disaster from the aspect of participatory sensing. Our POKEGA device enabled the rapid development of the world's first smartphone-connected radiation detector and accompanied by an inclusive community for radiation protection formed by voluntary experts and ordinary users.

We think that the POKEGA model will be useful in a wide range of applications, including environment monitoring and risk communication for potential hazards such as air, soil, and water contamination. Furthermore, in future efforts, we hope to be able to develop a similar weather sensor that allows data sharing for temperature, wind speed, and other inclement weather conditions. Another option, especially for Japan, would be a motion sensor device for smartphones to provide local real-time earthquake data over a wide area.

This paper proposed an interaction model for credible discussion by social inclusion between ordinary people and radiation experts in the community in the following three project development phases: start-up, collaboration, and mutual verification. Castelfranchi and Falcone proposed that trust is epistemically rational when based on well-motivated evidence and good inferences, and when credibility is correctly based on reliable sources, both external and internal [18]. To propagate appropriate risk communication based on rational trust, social inclusion is important for supporting the decisions of local residents by swiftly providing risk information regarding appropriate actions through discussions with experts.

Through an analysis of Twitter activity after the 2010 earthquake in Chile, Mendoza et al. demonstrated that the social media crowd has the potential to selfcorrect [19]. In addition, Starbird et al. examined the relationship between rumors and crowd correction on Twitter after the 2013 Boston Marathon Bombing [20], and $\mathrm{Oh}$ et al. found that information from credible sources suppresses anxiety through an analysis of 
Twitter data using both quantitative and qualitative methods following the 2010 earthquake in Haiti [21].

We assume that the collaborative process plays an important role in quick and wide-ranging monitoring in crisis management, and that the mutual verification process is effective for ensuring the reliability of instruments and fostering trust through cooperation and discussions between citizens and professionals via social media.

We developed Crowd Talks to characterize social media data based on MeCab, LDA, and PCA. As a result, we identified three major clusters of topics in the POKEGA Facebook community. According to cluster analysis, we discovered that POKEGA users needed rich content for disaster information and fixedpoint data sharing. The topic visualization method is considered to have great potential for future applications, not only for environmental monitoring, but also for other domains such as user-centered design and product safety.

\section{Acknowledgements}

This work has been conducted as a part of "Research Initiative on Advanced Software Engineering in 2015" supported by Software Reliability Enhancement Center (SEC), Information Technology Promotion Agency Japan (IPA).

\section{References}

[1] Y. Ishigaki, Y. Matsumoto, Y. Matsuno and K. Tanaka, "Participatory Radiation Information Monitoring with SNS after Fukushima", Short Paper - Social Media Studies, Proceedings of the ISCRAM 2015 Conference - Kristiansand, May 24-27, 2015

http://iscram2015.uia.no/wp-content/uploads/2015/05/8-1.pdf

[2] Y. Ishigaki, Y. Matsuno, K. Bando and K. Tanaka, "Environmental Health Communication and its visualization on Social Media - Case Study of Radiation Health Communication after the Fukushima Disaster -", "The full day Learning Health System Symposium: Designing and Generalizing the Challenge of the Learning Health System, Expanding the Community, and Maintaining Momentum', Hawaii International Conference on System Sciences (HICSS-49), January 5, 2016.

[3] G. Dearnaley and D. C. Northrop, "Semiconductor Counters for Nuclear Radiations", Second Edition, New York, John Wiley \& Sons, Ltd., 1966

[4] Y. Ishigaki, Y. Matsumoto, R. Ichimiya, K. Tanaka, "Development of Mobile Radiation Monitoring System
Utilizing Smartphone and Its Field Tests in Fukushima", IEEE SENSORS, Vol. 13, Issue 10, pp. 3520 -3526, 2013.

[5] T. Kuipers, C. V. Wout and F. Bader, "iPhone als stralingsdetector", Dutch Journal of Radiation Protection, Issue 2, pp. 32-34, 2011 (in Dutch).

[6] B. Van de Weijer, "Een onderzoeker in ieders broekzak Smartphones inhet regenwoud", Netherland, Volkskrant, 2013-07-13 (in Dutch).

[7] M. Mizoguchi, T. Ito and D. Kobayashi, "Environmental Monitoring of Village Contaminated by Radionuclides", Proc. AFITA/WCCA 2012, Taipei, Taiwan, Seminar(19)-03, 138, 2012.

[8] M. Turoff, B. Van de Walle, and S. R. Hiltz , "Information Systems for Emergency Management", 'Section 16: Emergency Response Information Systems: Past, Present, and Future', pp.369-388, London and New York, Routledge, 2010.

[9] K. Imai, "SPEEDI: a computer code system for the realtime prediction of radiation dose to the public due to an accidental release", Japan Atomic Energy Research Institute (JAERI) Report, No.1297, Vol.17, Issue 21, 1985.

[10] N. Onishi and M. Fackler, "Japan Held Nuclear Data, Leaving Evacuees in Peril", New York, The New York Times, Published: August 8, 2011.

[11] K. Ono, "Issues emerged after the 3.11 Earthquake in Japan focusing on risk governance deficits in radioactive materials: Deficits on risk assessment", '(booklet) Emerging Issues Learned from the 3.11 Disaster as Multiple Events of Earthquake, Tsunami and Fukushima Nuclear Accident', The Society for Risk Analysis Japan, pp.42-43, 2013.

[12] D. M. Blei, A. Y. Ng and M. I. Jordan, "Latent dirichlet allocation", the Journal of machine Learning research, 3 (2003): 993-1022.

[13] C. Sievert and K. E. Shirley, "LDAvis: A method for visualizing and interpreting topics", Proceedings of the Workshop on Interactive Language Learning, Visualization, and Interfaces, Baltimore, Maryland, USA, pp.63-70, 2014.

[14] N. Bal, M. Trudel, N. Richner, T. Ter-Stepanyan, "DoseNet Project to Develop a Dosimeter Network", Spring Semester, 2015 (Project report)

http://www.navrit.com/pdf/NE170-FinalReport.pdf

[15] JWP corporation (Press release), "Radiation Dosimeter for See-Camera", Sept 24, 2015

http://www.j-world.co.jp/pdf/radiation.pdf (Japanese)

[16] A. Segault, F. Tajariol, Y. Ishigaki and I. Roxin, "\#geiger2: Developing Guidelines for Radiation Measurements Sharing on Social Media", Short Paper Social Media Studies, Proceedings of the ISCRAM 2016 Conference - Rio de Janeiro, Brazil, May 2016 
[17] J.A. Burke, D. Estrin, M. Hansen, A. Parker, N. Ramanathan, S. Reddy and M. B. Srivastava, "Participatory Sensing", Proc. of the International Workshop on WorldSensor-Web (WSW'2006), ACM, October 31, Boulder, U.S.A., 2006.

[18] R. Falcone and C. Castelfranchi, "Trust and Deception in Virtual Societies", 'Chapter 3: Social Trust: A Cognitive Approach', Dordrecht , Springer Netherlands, pp. 55-90, 2001.

[19] M. Mendoza, B. Poblete and C. Castillo, "Twitter Under Crisis: Can we trust what we RT? ", 1st Workshop on Social Media Analytics (SOMA '10), July 25, 2010, Washington, DC, USA, ACM, pp. 71- 79.

http://snap.stanford.edu/soma2010/papers/soma2010_11.pdf

[20] K. Starbird, J. Maddock, M. Orand1 , P. Achterman and R. M. Mason, "Rumors, False Flags, and Digital Vigilantes: Misinformation on Twitter after the 2013 Boston Marathon Bombing" , iConference 2014 Proceedings, 2014-03-01, pp. 654-662, DOI: $10.9776 / 14308$

https://www.ideals.illinois.edu/bitstream/handle/2142/47257/ 308_ready.pdf?sequence $=2 \&$ isAllowed $=y$

[21] O. Oh, K. H. Kwon, H. R and Rao, "An Exploration of Social Media in Extreme Events: Rumor Theory and Twitter during the Haiti Earthquake 2010", ICIS 2010 Proceedings. Paper, 231, 2010. 